\title{
L'Educazione Continua in Medicina o educazione che manca?
}

\author{
Gianni Ascione ${ }^{1}$, Giuseppe Quintaliani² \\ ${ }^{1}$ Ufficio Formazione, Azienda Ospedaliera Perugia, Perugia \\ ${ }^{2} \mathrm{SC}$ Nefrologia, Azienda Ospedaliera di Perugia, Perugia
}

\begin{abstract}
Continuing medical education
Continuing medical education (CME) is an essential element of state-of-the-art medical practice. As it is structured today in most countries, CME must guarantee its own quality and independence. There should therefore be an independent professional body at the national level which is responsible for assessing and guaranteeing both quality and independence. This body will also have to oversee the participation of medical specialists in CME. A system of credits expresses the professional value of CME activities. Each activity is credited with a score, to be awarded to the participating specialist. CME should remain an ethical obligation that is subject to the disciplinary authority of the profession itself. It should be both an individual and collective obligation; in order to promote it and make it effective, each EM member state must provide the means of making CME available to all physicians. For the above reasons, $\mathrm{CME}$ is a strategic way to improve the quality of the health system.
\end{abstract}

Keywords: Continuing medical education, Improvement in professional standards, Quality in clinical management

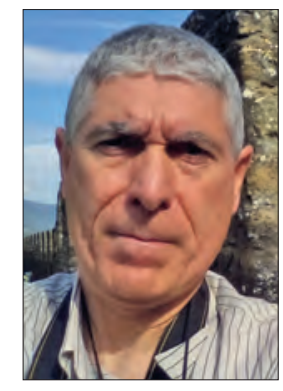

Gianni Ascione

\section{Che cos'è la EMC (Educazione Medica Continua)}

L'EMC è definita come un sistema per facilitare i cambiamenti nella pratica clinica e migliorarne la qualità. Questo sistema privilegia l'adeguamento delle competenze piuttosto che l'acquisizione generica di conoscenze. Scopo della EMC è il cambiamento delle pratiche cliniche obsolete e la diffusione delle nuove conoscenze rilevanti per la pratica clinica (Allegato 1).

Nella pagina iniziale del sito dei medici specialisti europei (1) si legge che la EMC é un obbligo e un dovere morale nei confronti dei pazienti. I pazienti hanno il diritto di aspettarsi che i medici siano in grado di somministrare la giusta cura secondo lo stato dell'arte.

Idealmente l'EMC è un processo di aggiornamento continuo delle conoscenze, che arricchisce e raffina l'esperienza clinica. La sola pratica clinica infatti non può essere sufficiente

Accepted: October 24, 2016

Published online: November 22, 2016

Indirizzo per la corrispondenza:

Dr. Giuseppe Quintaliani

Via Maturanzio, 31

06124 Perugia

g.quintaliani@yahoo.it perché l'esperienza individuale deve essere sottoposta a continui confronti con i risultati degli studi clinici diagnostici, prognostici e terapeutici che arricchiscono le basi conoscitive sulle quali si fonda la medicina moderna.

L'aggiornamento e la competenza devono risultare ben "visibili" ai pazienti e l'aggiornamento deve essere effettuato secondo regole e principi ben definiti.

In molti Paesi, sia europei che extraeuropei, sono stati avviati validi programmi di EMC; in altri sono in via di attuazione sotto la supervisione dell'European Accreditation Council for EMC (EACEMC) il cui scopo principale è rendere la EMC trasparente e ben focalizzata sui problemi clinici. I principi a cui ispirarsi sono:

- la EMC è un obbligo morale;

- la EMC è una responsabilità nazionale;

- le organizzazioni partecipanti usano, per uniformare l'unità di misura, "I'ora di EMC" (credit hour);

- tra le organizzazioni nazionali che partecipano a programmi di EMC vige il principio della reciprocità garantita.

I principi della EMC si sono andati sviluppando nel tempo e hanno portato, da parte della Standing Committee of European Doctors, alla formulazione della Dichiarazione di Dublino del 1993, che recepisce alcuni di questi criteri.

La EMC non è un mero aggiornamento compiuto in maniera sporadica e occasionale ma un vero e proprio "sistema" di valorizzazione della professione medica. I pazienti hanno fiducia nella professionalità dei medici che li curano e nella 
ALLEGATO 1 - La definizione di EMC prevista dalla legge

L'articolo 16-bis, D.L. 19 giugno 1999, n. 229 specifica: «... La formazione continua comprende l'aggiornamento professionale e la formazione permanente. L'aggiornamento professionale è l'attività successiva al corso di diploma..., diretta ad adeguare per tutto l'arco della vita professionale le conoscenze professionali. La formazione permanente comprende le attività finalizzate a migliorare le competenze e le abilità cliniche, tecniche e manageriali ed i comportamenti degli operatori sanitari al progresso scientifico e tecnologico con l'obiettivo di garantire efficacia, appropriatezza, sicurezza ed efficienza alla assistenza prestata dal Servizio sanitario nazionale».

loro capacità di garantire un servizio di interesse pubblico prioritario. Tale fiducia si basa sull'assunto che la professionalità medica pone in essere meccanismi in grado di identificare e correggere pratiche cliniche inefficaci e/o superate.

Per mantenere questo essenziale anello fiduciale che lega pazienti e medici è quindi necessario che tali meccanismi siano implementati dagli organi di governo della sanità, in cooperazione con organismi professionali; $i$ soli in grado di valutare in maniera appropriata la qualità della pratica professionale.

Un buon sistema, con garanzie di trasparenza sia per il pubblico che per i professionisti, è in grado di mantenere alti standard di qualità professionale e di meritare la fiducia degli utenti.

In Italia I'EMC è divenuta uno degli obiettivi strategici che il Piano Sanitario Nazionale (PSN) 2001/2003 ha proposto, affermando che il programma di formazione continua persegue il costante adeguamento al progresso scientifico e tecnologico delle competenze professionali e delle abilità cliniche e tecniche di tutti gli operatori sanitari, con la finalità di garantire efficacia, appropriatezza, sicurezza ed efficienza alle prestazioni sanitarie.

L'aggiornamento più proficuo parte dal confronto con $\mathrm{i}$ problemi clinici identificati nel contatto con il paziente: è qui che il medico prende coscienza della necessità di acquisire ulteriori conoscenze. I vantaggi dell'apprendimento basato su problemi sono numerosi: è più approfondito, aumenta e consolida l'esperienza, è più agevole perché ben focalizzato, promuove la collaborazione interdisciplinare, è più piacevole e aumenta la motivazione.

In un interessante editoriale apparso sul British Medical Journal (1) si afferma che la vecchia visione che il medico deve conoscere tutto e sapere tutto è sbagliata. "lo non so»: questa è la formula giusta per avere la spinta e la voglia di apprendere. L'apprendimento si basa sulla consapevolezza di non sapere e sulla capacità di trovare le opportune fonti di informazioni.

Il mezzo dell'aggiornamento è di cruciale importanza per ottenere buoni risultati (2) ma dipende dalle abitudini del singolo medico. In teoria, anche la sola lettura di letteratura medica può servire allo scopo, se perseguita con costanza e regolarità. Anche la lettura di riviste e libri, discussione di casi clinici e altri modi tradizionali di aggiornamento sono di qualche utilità; tuttavia, analisi dettagliate del valore di questo tipo di aggiornamento hanno evidenziato che esso ha un impatto scarso o nullo sui risultati (outcome) clinici (3).
L'aggiornamento più solido e produttivo è quello legato alla soluzione di problemi clinici. Quando un medico si confronta con un problema clinico - diagnostico, prognostico o terapeutico che sia - spesso emergono problemi per i quali egli non ha una risposta o per i quali non ha sufficienti conoscenze. II medico può risolvere i suoi dubbi in vari modi, dal consultare fonti di conoscenza a consultare colleghi esperti.

L'enorme espansione delle fonti di conoscenza attraverso internet e le possibilità di accesso alle stesse a costo molto basso sono un'opportunità unica per integrare l'aggiornamento del medico nella pratica clinica quotidiana (4). Telecomunicazioni sempre più rapide e strumenti portatili e user friendly come i palmtop, consentono al medico di consultare, al letto del malato o negli ambulatori, i grandi database della letteratura medica come PubMed e i manuali evidence-based di medicina interna e nefrologia come UpToDate. La rapida consultazione di questi database permette di ottenere risposte a specifici problemi clinici in tempi brevissimi, secondi o minuti e di stabilire, grazie a queste informazioni, decisioni cliniche tempestive, ben orientate e valide.

Purtroppo questi strumenti non sono ancora abbastanza diffusi nel nostro Paese e attualmente le strategie di ricerca delle informazioni sono poco conosciute dai medici. Su questo terreno, la formazione sulla "ricerca delle informazioni" può produrre risultati di grande portata.

Sfortunatamente a questo tipo di aggiornamento ancora non è stata data adeguata, concreta rilevanza dagli enti di accreditamento dell'EMC; anche se, in Italia, l'"applicazione nella pratica quotidiana dei principi e delle procedure dell' $e$ vidence based practice (EBM-EBN-EBP)" risulta formalmente inserita tra gli obiettivi di rilievo nazionale del sistema EMC.

Una delle possibili ragioni è che è difficilmente controllabile e verificabile. Tuttavia gli strumenti software e hardware per documentare l'accesso, l'uso e la durata dei contatti a fonti di conoscenza di alto valore (PubMed, Embase o manuali evidence based online, oltre a UpToDate) sono ora disponibili e le Società scientifiche hanno l'arduo compito di convincere gli organi di governo che investimenti in questo settore offrono un'opportunità senza pari per migliorare la qualità delle prestazioni professionali a costi bassi.

\section{Strategie di apprendimento}

Nella maggior parte dei casi il medico lavora a contatto con colleghi e con personale sanitario; la qualità della pra- 
tica medica dipende prima di tutto dal buon funzionamento dell'intero team (5).

È ben documentato che un ambiente di lavoro conflittuale e mal organizzato si riflette in maniera negativa sul processo della guarigione anche quando il trattamento clinico di per sé é ineccepibile.

Il tempo riservato alla EMC è importantissimo: i medici dovrebbero usufruire di periodi liberi da problemi clinici impegnativi, in modo da poter riflettere in maniera esauriente sulla necessità di cambiare strategie e approcci terapeutici.

La EMC ha poche possibilità di successo se viene svolta all'interno dell'orario di servizio, quando il medico è impegnato nella risoluzione di problemi complessi e impegnativi. In un ambiente in cui domina il conflitto e l'incertezza decisionale, è più rassicurante ancorarsi a pratiche inveterate (e spesso superate) piuttosto che introdurre approcci nuovi ma ancora poco conosciuti e praticati.

È innegabile che spesso i medici tendono ad adagiarsi sui risultati ottenuti e finiscono per aggiornarsi sempre meno. Nei sistemi sanitari maturi l'aggiornamento non è più discrezionale ma è parte di un sistema obbligatorio di riqualificazione professionale (6).

La EMC deve essere obbligatoria per rispondere alle necessità e agli standard sanitari dei vari servizi sanitari nazionali $(7,8)$. L'obbligatorietà tuttavia non è in contrasto con il principio che il singolo medico deve essere il protagonista nel controllo del processo. Infatti se il medico identifica l'area di miglioramento, per esempio, quella "toracica" potrà o continuare ad aggiornarsi nel suo campo o iniziare un altro processo di formazione a patto che sia lui a individuarne la necessità, anche all'esterno della propria specialità (il chirurgo molto esperto di chirurgia, disegna il processo, lo persegue, ne valuta il risultato e mantiene la responsabilità di ridefinire il processo se necessario). Ciò configura un vero e proprio processo di PDSA (Plan-Do-Study-Check-Act): Programma, Fai, Valuta, Agisci (9).

\section{L'EMC come strumento formativo e strumento strategico aziendale}

La EMC come abbiamo visto, parte dal presupposto che ogni singolo professionista valuti le proprie carenze professionali e vi ponga rimedio con un adeguato aggiornamento. Ciò è non solo corretto ma auspicabile. Tuttavia, poiché la EMC è anche obbligatoria e impegna importanti risorse economiche, potrebbe essere utilizzata come strumento formativo a disposizione delle organizzazioni che, ponendone l'onere a loro carico, la utilizzino come strumento strategico.

Abbiamo già ricordato infatti che scopo della EMC è il cambiamento delle pratiche cliniche obsolete e la diffusione delle nuove conoscenze rilevanti per la pratica clinica; concetto che è alla base dei programmi di formazione delle organizzazioni sanitarie, in cui la conoscenza viene data per acquisita dai corsi universitari e certificata al momento del concorso di assunzione, mentre l'adeguamento delle pratiche cliniche obsolete è ancora un grosso problema strutturale.

È questo un concetto che ancora stenta a divenire un "sistema" ma che dovrà comunque portare a una nuova gestione della formazione e dell'aggiornamento all'interno dei nostri ospedali.

Nel sito del Ministero si può già leggere quali sono gli obiettivi strategici della EMC, obiettivi formativi di interesse nazionale che sono stati stabiliti dalla conferenza permanente per i rapporti tra lo Stato le Regioni e le Province autonome (10).

Lo stesso percorso dovrebbe essere seguito anche dalle singole Aziende ospedaliere, che potrebbero sviluppare programmi EMC strategici per la formazione dei propri operatori. Tipici esempi sono lo sviluppo di conoscenze sul consenso informato, sulla legge sulla privacy, sulla comunicazione in senso lato, sull'uso delle risorse informatiche, sulla EBM, sul management sanitario.

Questi corsi servono, e sono serviti, non solo a coprire le esigenze formative di un gran numero di professionisti ma anche ad omogeneizzare alcuni problemi a livello aziendale permettendo all'alta direzione di dirigere le risorse formative dove ce ne fosse più bisogno per colmare lacune che difficilmente avrebbero potuto così capillarmente e uniformemente raggiungere tutte le figure professionali all'interno delle singole aziende, senza il pungolo dell'EMC.

A questo proposito, lo sviluppo dell'EMC come assetto strategico per implementare nuove conoscenze, è uno dei punti cardine dello sviluppo delle risorse umane anche all'interno delle singole Strutture Complesse. I responsabili delle Strutture Complesse potrebbero (e dovrebbero) infatti utilizzare l'EMC come strumento di programmazione e di sviluppo del capitale umano, implementando un sistema di Knowledge Management.

È ben noto che la frequenza a corsi, congressi, studi e trial è un buon strumento di crescita culturale. Spesso, però, il sistema viene lasciato a se stesso, con criteri di rotazione o di sviluppo di interessi singoli. Sarebbe opportuno che un buon programma EMC comportasse una visione strategica sul ruolo della formazione e dell'aggiornamento.

Lo strumento EMC permetterebbe, se gestito come una risorsa e una opportunità, di sviluppare e implementare progetti e programmi clinici di indubbio spessore professionale. I programmi di accreditamento e certificazione prevedono infatti che la formazione debba essere attuata secondo uno schema e con criteri noti, condivisi e formalizzati. L'organizzazione è quindi tenuta a formare in conformità ai suoi interessi e scopi, nell'ambito di una visione strategica della risorsa "formazione".

Questo concetto apre alcuni dibattiti sul significato della EMC e sui costi per ottenere i crediti.

In altre parole, un professionista potrebbe aver colmato il suo debito con un programma EMC organizzato dalla propria azienda, magari su competenze informatiche ma non aver colmato proprie lacune professionali. Nel primo caso, a 
fronte di un punteggio EMC assolto del tutto gratuitamente, si ha la non scelta e quindi il perdurare di lacune professionali. Nel secondo caso, per colmare le lacune professionali, il professionista dovrebbe pagarsi di tasca propria le spese dell'aggiornamento. È questo un punto critico di discussione, che coinvolge gli Ordini professionali, le Aziende sanitarie, i singoli professionisti.

Uno dei sistemi che potrebbe aiutare a dirimere la questione e da poter utilizzare per l'aggiornamento individuale è la FAD (Formazione a Distanza). Il sistema, in vigore in quasi tutti i Paesi dove sono stati sviluppati programmi EMC, permette il raggiungimento di crediti EMC con sistemi che possono andare dal rispondere a quiz sulle riviste a cui il singolo è abbonato, a programmi di conferenze online come nel caso di Hypertension, Dialysis and Clinical Nephrology (HDCN) -NDT educational. II Ministero ha intenzione di sviluppare fortemente questo aspetto con l'obiettivo di portare la FAD a una percentuale di circa l' $80 \%$ di tutta la EMC.

Appare comunque chiaro che, qualunque sia la scelta delle organizzazioni chiamate a svolgere programmi di EMC, è necessario riservare e ricavare uno spazio all'attività di apprendimento da effettuare nell'ambiente di lavoro. Infatti è doveroso ricordare che l'aggiornamento, inteso come risorsa strategica per il miglioramento continuo, ha trovato uno spazio anche nel Contratto collettivo nazionale di lavoro (CCNL) dei sanitari che riserva loro quattro ore (riducibili a tre) per l'aggiornamento continuo. Tali ore sono: a) obbligatorie; b) non possono essere utilizzate per la normale attività clinica; c) sono cumulabili e spendibili oltre gli otto giorni di permesso per studio. Norme e specifiche sono rintracciabili nell'ottimo sito di management sanitario del Professor Scheggi (11) (Allegato 2).

\section{II programma europeo dei medici specialisti per l'aggiornamento in Nefrologia (UEMS)}

L'Union Européenne des Médecins Spécialistes (UEMS) è l'organismo internazionale che, dal 1999, ha sviluppato un sistema di accreditamento europeo per la formazione in sanità, istituendo l'European Accreditation Council for Continuing Medical Education (EACCME ${ }^{\oplus}$ ).

L'UEMS ha sviluppato delle raccomandazioni per la EMC in Nefrologia. Le specifiche possono essere consultate sul sito della organizzazione (12). Qui basterà ricordare che sono state sviluppate diciassette raccomandazioni, che il ciclo formativo viene posto in cinque anni, che si propone un sistema formativo per crediti come quello vigente negli USA con due tipi di crediti e un minimo obbligatorio di ore-crediti. In Europa e più in generale a livello internazionale l'EMC è in stadi di sviluppo molto diversi.

In Italia, II decreto legislativo 30 dicembre 1992, n. 502, di riforma in senso aziendale della sanità, come integrato dal decreto legislativo 19 giugno 1999, n. 229, ha istituzionalizzato anche nel nostro Paese l'EMC, denominandola Educazione Continua in Medicina (ECM); in questo modo è stato istituito l'obbligo della formazione continua per i professionisti della sanità, medici e non.

L'accordo Stato-Regioni del 2009 definisce l'ECM “L'attività educativa che serve a mantenere, sviluppare e incrementare le conoscenze, le competenze e le performance degli operatori della sanità" (13).

Dal punto di vista organizzativo, l'ECM si configura come un sistema complesso, con una governance articolata e distribuita tra diversi organismi:

- $\quad$ il Ministero della Salute;

- la Commissione nazionale per la formazione continua;

- le Regioni e le Province autonome di Trento e Bolzano;

- $\quad$ gli ordini e collegi professionali;

- gli organizzatori di eventi formativi d'interesse per il progetto ECM (Provider);

- $\quad$ i professionisti sanitari cui è destinata l'offerta formativa;

- le Società scientifiche;

- le associazioni professionali e le organizzazioni sindacali;

- Il Consorzio Gestione Anagrafica Professioni Sanitarie (COGEAPS).

Con accordi Stato-Regioni vengono, fra l'altro definiti e aggiornati periodicamente la programmazione di Formazione Continua in Medicina, gli obiettivi, l'organizzazione e le regole per la governance del sistema ECM.

Dal $1^{\circ}$ gennaio 2008, con l'entrata in vigore della Legge 24 dicembre 2007, n. 244, la gestione amministrativa del programma di ECM e il supporto alla Commissione Nazionale per la Formazione Continua, sono stati trasferiti dal Ministero della Salute all'Agenzia nazionale per i servizi sanitari regionali (AGENAS) (14).

Vediamo in breve come è organizzato e come funziona concretamente il sistema.

Attualmente il sistema si configura su due livelli, nazionale e regionale. I fornitori di formazione (Provider), per poter operare come tali, devono accreditarsi presso uno dei due sistemi. L'accreditamento "è il riconoscimento, da parte degli organismi nazionali o regionali deputati, che un soggetto è attivo e qualificato nel campo della formazione continua in sanità e che pertanto è abilitato a realizzare attività formative idonee per l'ECM, individuando e attribuendo direttamente i crediti formativi ai partecipanti" (15).

Gli enti accreditanti sono, a livello nazionale, la Commissione nazionale per la formazione continua e, a livello regionale, le Regioni e le Province autonome.

Le Aziende sanitarie e altri soggetti erogatori di prestazioni sanitarie pubblici o privati ed eventuali enti di formazione a partecipazione prevalente pubblica regionale o provinciale, sono accreditati dalle Regioni, Province autonome o Enti preposti. Gli altri enti e istituzioni pubblici e privati, ivi compresi gli Ordini e Collegi professionali e le Società scientifiche, sono 
ALLEGATO 2 - Norme legislative

CCNL 2002_2005

ARTICOLO 14

orario di lavoro dei dirigenti

\section{Quattro ore riservate ad attività non assistenziale}

Nello svolgimento dell'orario di lavoro previsto per i dirigenti medici e veterinari, quattro ore dell'orario settimanale sono destinate ad attività non assistenziali, quali l'aggiornamento professionale, l'ECM, la partecipazione ad attività didattiche, la ricerca finalizzata. Tale riserva di ore non rientra nella normale attività assistenziale, non può essere oggetto di separata ed aggiuntiva retribuzione. Essa va utilizzata di norma con cadenza settimanale ma, anche per particolari necessità di servizio, può essere cumulata in ragione di anno per impieghi come sopra specificati ovvero infine utilizzata anche per l'aggiornamento facoltativo in aggiunta agli otto giorni l'anno di permesso retribuito per la partecipazione a convegni, congressi, corsi di aggiornamento facoltativi previsti dall'articolo 23, comma 1, del CCNL 5 dicembre 1996. Tale riserva va resa in ogni caso compatibile con le esigenze funzionali della struttura di appartenenza e non può in alcun modo comportare una mera riduzione dell'orario di lavoro.

\section{Accordo Stato-Regioni del $1^{\circ}$ agosto 2007}

«Perché sia efficace la formazione continua non deve svilupparsi con modalità occasionali, ma secondo percorsi formativi finalizzati al raggiungimento di obiettivi coerenti con lo sviluppo professionale individuale e con le priorità, le innovazioni e le strategie del sistema sanitario, a livello locale, regionale e nazionale», e ancora «Il professionista della sanità ha il diritto-dovere di acquisire crediti ECM su tematiche coerenti con il proprio lavoro. L'aggiornamento, pertanto, dovrà essere finalizzato sia ad obiettivi scelti sulla base della specificità professionale, sia ad obiettivi di interesse generale legati alla programmazione sanitaria.»

\section{Il comma 1 dell’articolo 16-quater del decreto legislativo n. 502}

La formazione continua costituisce per le Aziende sanitarie un preciso dovere al quale non possono sottrarsi, e nel quale devono investire risorse adeguate (pari almeno all'1\% del monte salari).

Il comma 1 dell' articolo 16-bis del decreto legislativo n. 502

La formazione continua (che comprende l'aggiornamento professionale e la formazione permanente), consiste in attività di qualificazione specifica per i diversi profili professionali, attraverso la partecipazione a corsi, convegni, seminari organizzati da istituzioni pubbliche o private accreditate ai sensi del presente decreto, nonché soggiorni di studio e la partecipazione a studi clinici controllati e ad attività di ricerca, di sperimentazione e di sviluppo.

accreditati, di regola, a livello nazionale.

I Provider accreditati assegnano i crediti ECM a ciascun evento formativo, in base ai criteri definiti a livello nazionale e regionale; attestano inoltre ai partecipanti agli eventi i crediti conseguiti, una volta accertato l'apprendimento. Tali crediti hanno valore su tutto il territorio nazionale.

Gli accordi Stato-Regione e la Commissione nazionale per la formazione continua, hanno stabilito in 150 crediti il debito formativo complessivo, computato su base triennale (ultimo triennio, 2014-2016), in ragione di 50 crediti per anno, con un minimo di 25 e un massimo di 75 crediti conseguibili in un anno. Sono stati poi previsti meccanismi di esonero o esenzione totale o parziale e di riduzione dell'obbligo dei crediti (16).

Recentemente, infine, sono state estese ai professionisti in rapporto di dipendenza, le disposizioni già vigenti per i liberi professionisti, circa la possibilità di acquisire annualmente $i$ crediti formativi in maniera flessibile, fermo rimanendo il rispetto del monte crediti su base triennale (150 crediti) (17).

I Provider accreditati comunicano i crediti assegnati, all'ente accreditante e al COGEAPS, (Consorzio Gestione Anagrafica Professioni Sanitarie) (18), che gestisce l'anagrafe centralizzata dei crediti ECM, a beneficio degli Ordini, Collegi, Associazioni professionali e dei professionisti della sanità.

Sul piano qualitativo, il sistema prevede la definizione di obiettivi formativi nazionali (a cura della Commissione nazionale per la formazione continua) e regionali, quale strumento per orientare i programmi di formazione in sanità e definire le adeguate priorità nell'interesse del Sistema Sanitario Nazionale (SSN).

Gli obiettivi trovano poi articolazione e sviluppo nel piano formativo individuale (cd Dossier formativo) o di équipe, in cui assumono rilievo tre tipologie di obiettivi:

- tecnico professionali, finalizzati allo sviluppo di competenze e conoscenze dello specifico professionale, attraverso eventi formativi rivolti alla professione e/o disciplina di appartenenza;

- $\quad$ di processo, correlati alla formazione sui processi di produzione delle attività sanitarie, per promuoverne il miglioramento di qualità, efficienza, efficacia e appropriatezza;

- di sistema, correlati alla formazione interprofessionale, sui sistemi sanitari.

\section{Il futuro e le attività formative a distanza}

È innegabile che il futuro corre sulle linee dell'informazione elettronica e a nessuno sfugge che sul web si è già stabilita una base conoscitiva in continuo rinnovamento per 
tutte le attività direttamente e indirettamente connesse alla medicina e più in generale ai problemi della salute.

Attualmente i congressi e i seminari sono una fonte rilevante dell'aggiornamento medico ed è plausibile immaginare che queste modalità, "analogiche", di erogazione dell'informazione, rimarranno residuali. E ciò considerando che i punteggi assegnati a tali eventi sono del tutto residuali, come forse è giusto che sia, sia per la enorme varietà di argomenti trattati, sia per la presenza di sessioni parallele, sia per il poco tempo di apprendimento, sia per la dubbia possibilità di accertare quanto imparato (i test non sono certo un metodo affidabile al $100 \%$, per valutare l'apprendimento).

Già ora si delineano nuove modalità e strumenti dell'aggiornamento medico basati su siti educazionali e newsletter in continuo arricchimento.

$\mathrm{Ne}$ sono esempio paradigmatico alcune iniziative di grande impatto, realizzate nell'ambito delle malattie renali e dell'ipertensione arteriosa: Hypertension Dialysis and Clinical Nephrology (HDCN) (http//www.hdcn.com) e Nephrology, Dialysis and Transplantation-educational for kidney and blood pressure disorders (NDT-E) (http://www.ndt-educational.org/) oltre a UpToDate, (www.uptodate.com).

Queste risorse educazionali elettroniche offrono un vasto panorama di opzioni di aggiornamento: presentazioni scientifiche congressuali in forma di diapositive commentate (commento scritto e/o audio), registrazioni audio di letture congressuali, segnalazioni di articoli scientifici di alto impatto clinico, articoli ripresi integralmente dalle principali riviste di settore.

Alcune presentazioni e alcuni articoli sono accompagnati da una serie articolata di quesiti che consentono un'autoverifica dell'apprendimento. Mentre HDCN è una risorsa CME riconosciuta che eroga da circa quattro anni crediti riconosciuti negli USA, in Europa non esiste ancora un riconoscimento ufficiale per le risorse educazionali che operano via internet.

Il successo di queste risorse elettroniche è crescente. HDCN ha oltre 24.000 abbonati (13.000 dei quali hanno accesso a pagamento al sito in versione completa) mentre NDT-E sta registrato una continua espansione dell'utenza e ha superato i 10.000 contatti/mese.

NDT-E è una risorsa matura per erogare web-education in Nefrologia in Europa e il suo impatto potrà crescere in maniera ancor più spiccata quando l'UEMS riconoscerà i crediti distribuiti per via elettronica.

UpToDate è partner del sistema di aggiornamento continuo americano e utilizzato da diverse Società scientifiche. UpToDate è anche un libro edito solo online per permetterne l'aggiornamento continuo tanto che si trovano topic aggiornati da 15, 20 giorni massimo un mese. È quindi un formidabile e autorevole strumento di aggiornamento e di consultazione per i medici che abbiano dubbi, interrogativi, curiosità e necessità di avere informazioni attendibili e accurate in tempi brevi o per studio. Sarà forse per questo che alcuni amministratori di alto livello hanno deciso di non rinnovarne l'abbonamento per risparmiare, come se il miglior risparmio non fosse fare bene il medico.

Nel nostro Paese, il sistema di Educazione Continua in Medicina ha, solo di recente, trovato una certa compiutezza organizzativa e regolamentare. Ritarda, invece, lo sviluppo del profilo qualitativo e selettivo della formazione in medicina; quello che, oltre a precostituire un generico debito formativo da colmare, qualifichi il debito anche in funzione delle conoscenze, competenze e abilità correlate alla professionalità e alla relativa disciplina, come richiesto dal Dossier formativo.

Un nefrologo, per esempio, potrebbe oggi soddisfare interamente il fabbisogno di crediti formativi con corsi di informatica, sul diritto, sulla sicurezza, sull'anticorruzione o sullo smaltimento rifiuti; senza alcun obbligo di formazioneaggiornamento su argomenti clinici, magari pertinenti alla propria disciplina.

Il sistema si popola, così, di storture che capovolgono il significato stesso di "aggiornamento". Alcune ditte del farmaco sponsorizzano FAD spesso "drogate" ma appetibili perché consentono l'aggiornamento. D'altro canto, i sindacati sono spesso conniventi, perché il sistema consente a tutti di ottenere gli agognati crediti ma senza un vero costrutto clinico.

Una tendenza, questa, per esperienza presente anche nelle Aziende sanitarie; laddove si tende a prediligere argomenti di carattere generale e non sempre clinici, purché inclusivi di tutte le professionalità sanitarie, a scapito del mantenimento e aggiornamento delle competenze cliniche specifiche.

Alcune organizzazioni professionali, come i dietisti ANDID, si sono dati, a riguardo, regole più stringenti; considerando, per esempio, obbligatorio conseguire almeno metà dei crediti ECM su temi pertinenti alla disciplina nefrologica. Ciò per potersi vedere riconosciuta la qualificazione di dietista renale.

E ancora peggio le regole attuali non ti riconoscono crediti formativi attraverso l'accesso all'ECM online di riviste prestigiose come NEJM, Lancet, BMJ; mentre tale riconoscimento sussiste, se vai a congressi dove un relatore ti racconta cosa è stato pubblicato sul NEJM, Lancet eccetera.

Un assurdo, quindi, quello di un sistema che ha lasciato indietro lo sviluppo del suo profilo più pregnante, dando la stura a contraddizioni non ancora risolte; giacché, dal lato opposto, si parla e legifera sempre più insistentemente su temi quali appropriatezza, risparmio, uso oculato dei percorsi diagnostici e terapeutici assistenziali (PDTA) e addirittura ospedale per intensità di cura.

È esperienza ricorrente in ospedale che il numero di consulenze richieste agli specialisti sia enorme e che molti specialisti si comportino in maniera difforme. Anche perché i PDTA sono focalizzati sulle realtà locali e non su quello che è la best practice o che si dovrebbe fare secondo le ultime conoscenze. Ricordo solo che il nuovo decreto sulla responsabilità medica tiene e terrà sempre più in deciso conto le Linee guida e su queste i medici saranno chiamati a rispondere e a difendersi, 
non certo sui crediti ECM ottenuti parlando di informatica, dei rifiuti o degli incendi in ospedale.

Insomma, sarebbe opportuno che se il ministro o gli assessori regionali volessero dei medici aggiornati per curare bene, in maniera appropriata, giusta ed efficace, tenessero più in conto l'aggiornamento scientifico cercando di soddisfare l'assioma dell'ECM che, lo ripetiamo, è un sistema per facilitare i cambiamenti nella pratica clinica e migliorarne la qualità e non fare i crediti ECM come se fossero i punti di un supermercato.

Sarà forse per questo che attualmente il sistema dell'ECM ha delle grosse défaillance: a due mesi dalla scadenza del triennio formativo sono in regola il $47 \%$ dei dottori (20). Infettivologi (il 59\% ha già raggiunto i 150 crediti), medici di famiglia e pediatri (58\%) i più diligenti. Ortopedici e chirurghi i meno attenti all'obbligo: il $27 \%$ dichiara di aver conseguito meno di 50 crediti. La FAD si conferma modalità di fruizione prediletta dai camici bianchi (48\%) (19).

Occorre quindi imprimere un'accelerazione importante allo sviluppo del profilo qualitativo del sistema dell'Educazione Continua in Medicina. E ciò, sia enfatizzando il ricorso alla FAD e agli strumenti più qualificanti di divulgazione della conoscenza tecnico-professionale specifica e dell'apprendimento online (riviste, banche dati evidence based, per esempio), sia portando a regime il dossier formativo, col supporto di precisi vincoli che obblighino all'implementazione di tutte e tre le tipologie di obiettivi previsti, a partire da quelli tecnico-professionali.

\section{Disclosures}

Financial support: No financial support was received for this submission.

Conflict of interest: The authors have no conflict of interest.

\section{Bibliografia}

1. https://www.uems.eu/areas-of-expertise/cme-cpd.

2. "I don't know": the three most important words in education. BMJ. 1999;318:0.

3. How best to learn BMJ. 2000;320:a. doi: http://dx.doi.org/ 10.1136/bmj.320.7232.0/a.

4. Holm HA. Should doctors get CME points for reading? BMJ. 2000;320(7232):394-5.
5. LaPorte RE, Linkov F, Villasenor T, et al. Papyrus to PowerPoint (P 2 P): metamorphosis of scientific communication. BMJ. 2002;325(7378):1478-81.

6. du Boulay C. From CME to CPD: getting better at getting better? BMJ. 2000;320(7232):393-4.

7. Davis DA, Thompson MA, Oxman AD, Haynes RB. Changing physician performance: a systematic review of the effect of continuing medical education strategies. JAMA. 1995;274(9):700-5.

8. Chastonay P, Brenner E, Peel S, Guilbert FF. The need for more efficacy and relevance in medical education. Med Educ. 1996;30(4):235-8.

9. Scally G, Donaldson LJ. The NHS's 50 anniversary. Clinical governance and the drive for quality improvement in the new NHS in England. BMJ. 1998;317(7150):61-5.

10. Sharon J. Building a learning organization: communities of practice, self directed learning and continuing Medical Education. The Journal of Continuing Education in the Health Professions. 1997; 17:5-11.

11. http://www.salute.gov.it/portale/temi/p2_4.jsp?area=ecm.

12. http://www.health-management.it/.

13. https://www.uems.eu/uems-activities/accreditation/eaccme

14. Accordo Stato-Regioni del 5 novembre 2009, Allegato A, pag. 1. https://www.inrca.it/inrca/files/FORMAZIONE/ACCORDO_5_ NOVEMBRE_2009.pdf.

15. http://ape.agenas.it/ecm/ecm.aspx.

16. Regolamento applicativo dei criteri oggettivi di cui all'accordo Stato-Regioni del 5 novembre 2009, per l'accreditamento, approvato dalla Commissione nazionale per la formazione continua il 13 gennaio 2010. http://ape.agenas.it/documenti/1. regolamento_provider.pdf.

17. Determina della CNFC del 17 luglio 2013 "Esoneri, Esenzioni, Tutoraggio Individuale, Formazione all'estero, Autoapprendimento, Modalità di registrazione e certificazione". http://ape. agenas.it/comunicati/comunicati.aspx?ID $=40$.

18. Determina della CNFC del 23/07-10/10/2016 in materia di crediti formativi ECM. http://ape.agenas.it/documenti/normativa/delibera_CNFC_7_luglio_2016.pdf.

19. Il Cogeaps è l'organismo nazionale deputato alla gestione delle anagrafiche nazionali e territoriali, dei crediti ECM attribuiti ai professionisti che fanno capo agli Ordini, Collegi nonché le rispettive Federazioni nazionali e Associazioni professionali; consentendo a questi le relative funzioni di certificazione delle attività formative svolte. http://www.cogeaps.it/Cogeaps/10/11/Il+Consorzio+CoGeAPS.html.

20. Quotidiano sanità 2 novembre 2016 ECM. Più della metà dei medici non è in regola. $E$ tra 2 mesi scade il triennio formativo. L'indagine. Quotidiano sanità 2/11/2016. http://www.quotidianosanita.it/studi-e-analisi/articolo.php?articolo_id=44607. 